\title{
Convergence theorem for common fixed points of a finite family of multi-valued Bregman relatively nonexpansive mappings
}

Naseer Shahzad ${ }^{1 *}$ and Habtu Zegeye ${ }^{2}$

"Correspondence:

nshahzad@kau.edu.sa

${ }^{1}$ Department of Mathematics, King

Abdulaziz University, P.O. Box 80203

Jeddah, 21589, Saudi Arabia

Full list of author information is

available at the end of the article

\begin{abstract}
In this paper, it is our purpose to introduce an iterative process for the approximation of a common fixed point of a finite family of multi-valued Bregman relatively nonexpansive mappings. We prove that the sequence of iterates generated converges strongly to a common fixed point of a finite family of multi-valued Bregman nonexpansive mappings in reflexive real Banach spaces.

MSC: 47H05; 47H09; 47H10; 47J25; 49J40; $90 \mathrm{C} 25$
\end{abstract}

Keywords: Bregman projection; Legendre function; multi-valued Bregman nonexpansive mapping; relatively nonexpansive multi-valued mapping; single-valued Bregman nonexpansive mapping; strong convergence

\section{Introduction}

Let $E$ be a reflexive real Banach space $E$, and $E^{*}$ its dual. Let $f: E \rightarrow(-\infty, \infty]$ be a proper convex and lower semicontinuous function. The subdifferential off at $x \in E$ is the convex set defined by

$$
\partial f(x)=\left\{x^{*} \in E^{*}: f(x)+\left\langle x^{*}, y-x\right\rangle \leq f(y), \forall y \in E\right\} .
$$

The Fenchel conjugate of $f$ is the function $f^{*}: E^{*} \rightarrow\left(-\infty,+\infty\right.$ ] defined by $f^{*}(y)=$ $\sup \{\langle y, x\rangle-f(x): x \in E\}$. It is not difficult to check that when $f$ is proper and lower semicontinuous, so is $f^{*}$.

The function $f$ is said to be essentially smooth if $\partial f$ is both locally bounded and singlevalued on its domain. It is called essentially strictly convex, if $(\partial f)^{-1}$ is locally bounded on its domain and $f$ is strictly convex on every convex subset of dom $\partial f . f$ is said to be Legendre, if it is both essentially smooth and essentially strictly convex.

Let $\operatorname{dom} f=\{x \in E: f(x)<\infty\}$. Then for any $x \in \operatorname{int}(\operatorname{dom} f)$ and $y \in E$, the right-hand derivative of $f$ at $x$ in the direction of $y$ is defined by

$$
f^{\circ}(x, y):=\lim _{t \rightarrow 0^{+}} \frac{f(x+t y)-f(x)}{t} .
$$

If the limit in (1.2) exists then $f$ is called Gâteaux differentiable at $x$. In this case, $f^{\circ}(x, y)$ coincides with $\nabla f(x)$, the value of the gradient $\nabla f$ of $f$ at $x$. The function $f$ is called Gâteaux

\section{严 Springer}

@2014 Shahzad and Zegeye; licensee Springer. This is an Open Access article distributed under the terms of the Creative Commons Attribution License (http://creativecommons.org/licenses/by/2.0), which permits unrestricted use, distribution, and reproduction in any medium, provided the original work is properly cited. 
differentiable if it is Gâteaux differentiable for any $x \in \operatorname{int}(\operatorname{dom} f)$. The function $f$ called Fréchet differentiable at $x$ if the limit in (1.2) is attained uniformly for all $y \in E$ such that $\|y\|=1$ and $f$ is said to be uniformly Fréchet differentiable on a subset $C$ of $E$ if the limit is attained uniformly for $x \in C$ and $\|y\|=1$. When the subdifferential of $f$ is single-valued, it coincides with the gradient $\partial f=\nabla f$ (see [1]).

We remark that if $E$ is a reflexive Banach space. Then we have

(1) $f$ is essentially smooth if and only if $f^{*}$ is essentially strictly convex (see [2], Theorem 5.4).

(2) $(\partial f)^{-1}=\partial f^{*}$ (see [3]).

(3) $f$ is Legendre if and only if $f^{*}$ is Legendre (see [2], Corollary 5.5).

(4) If $f$ is Legendre, then $\nabla f$ is a bijection satisfying $\nabla f=\left(\nabla f^{*}\right)^{-1}$, $\operatorname{ran} \nabla f=\operatorname{dom} \nabla f^{*}=\operatorname{int}\left(\operatorname{dom} f^{*}\right)$ and $\operatorname{ran} \nabla f^{*}=\operatorname{dom} \nabla f=\operatorname{int}(\operatorname{dom} f)$ (see [2],

Theorem 5.10).

When $E$ is a smooth and strictly convex Banach space, one important and interesting example of Legendre function is $f(x):=\|x\|^{p}(1<p<\infty)$. In this case the gradient $\nabla f=p J_{p}$ $(1<p<\infty)$, where $J_{p}$ is the generalized duality mapping from $E$ into $2^{E^{*}}$ defined by

$$
J_{p}(x)=\left\{f^{*} \in E^{*}:\left\langle x, f^{*}\right\rangle=\|x\|^{p},\left\|f^{*}\right\|=\|x\|^{p-1}\right\} .
$$

In particular, $J=J_{2}$ is called the normalized duality mapping. It is well known that if $E^{*}$ is strictly convex, then $J_{p}$ is single-valued and that

$$
J_{p}(x)=\|x\|^{p-2} J(x), \quad x \neq 0 .
$$

If $E=H$, a Hilbert space, then $J$ is the identity mapping and hence $\nabla f=2 I$, where $I$ is the identity mapping in $H$.

In this paper, $E$ is a reflexive real Banach space, $f: E \rightarrow(-\infty,+\infty]$ is a proper, lower semicontinuous, and convex function, and $f^{*}: E^{*} \rightarrow(-\infty,+\infty]$ is the Fenchel conjugate of $f$.

Let $f: E \rightarrow(-\infty,+\infty]$ be a Gâteaux differentiable function. The function $D_{f}: \operatorname{dom} f \times$ $\operatorname{int}(\operatorname{dom} f) \rightarrow[0,+\infty)$ defined by

$$
D_{f}(x, y):=f(x)-f(y)-\langle\nabla f(y), x-y\rangle
$$

is called the Bregman distance with respect to $f[4]$. Since $(\nabla f)^{-1}=\nabla f^{*}$ and $f^{*}(\nabla f)=$ $\langle x, \nabla f(x)\rangle-f(x)$, it is easy to check that

$$
D_{f^{*}}(\nabla f(y), \nabla f(x))=D_{f}(x, y) .
$$

A Bregman projection [5] of $x \in \operatorname{int}(\operatorname{dom} f)$ onto the nonempty closed and convex set $C \subset \operatorname{int}(\operatorname{dom} f)$ is the unique vector $P_{C}^{f}(x) \in C$ satisfying

$$
D_{f}\left(P_{C}^{f}(x), x\right)=\inf \left\{D_{f}(y, x): y \in C\right\}
$$

Remark 1.1 If $E$ is a smooth and strictly convex Banach space and $f(x)=\|x\|^{2}$ for all $x \in E$, then we have $\nabla f(x)=2 J x$, for all $x \in E$, where $J$ the normalized duality mapping and hence 
the function $D_{f}(x, y)$ reduces to $\phi(x, y)$ which is defined by $\phi(x, y)=\|x\|^{2}-2\langle x, J y\rangle+\|y\|^{2}$ for all $x, y \in E$, which is the Lyapunov function introduced by Alber [6], and $P_{C}^{f}(x)$ reduces to the generalized projection $\Pi_{C}(x)$ (see, e.g., [6]), which is defined by

$$
\phi\left(\Pi_{C}(x), x\right)=\min _{y \in C} \phi(y, x)
$$

If $E=H$, a Hilbert space, then $J$ is the identity mapping and hence the Bregman distance becomes $D_{f}(x, y)=\|x-y\|^{2}$, for $x, y \in H$, and the Bregman projection $P_{C}^{f}(x)$ reduces to the metric projection $P_{C}$ of $H$ on to $C$.

Let $C$ be a nonempty closed and convex subset of $\operatorname{int}(\operatorname{dom}(f))$. Let $T: C \rightarrow \operatorname{int}(\operatorname{dom}(f))$ be a mapping. An element $p \in C$ is called a fixed point of $T$ if $T(p)=p$. The set of fixed points of $T$ is denoted by $F(T)$. A point $p$ in $C$ is said to be an asymptotic fixed point of $T$ (see [7]) if $C$ contains a sequence $\left\{x_{n}\right\}$ which converges weakly to $p$ such that $\lim _{n \rightarrow \infty} \| x_{n}-$ $T x_{n} \|=0$. The set of asymptotic fixed points of $T$ will be denoted by $\widehat{F}(T) . T$ is said to be nonexpansive if $\|T x-T y\| \leq\|x-y\|$ for each $x, y \in C$, and is called quasi-nonexpansive if $F(T) \neq \emptyset$ and $\|T x-p\| \leq\|x-p\|$ for all $x \in C$ and $p \in F(T)$. The mapping $T$ is called relatively nonexpansive if (A1) $F(T) \neq \emptyset$; (A2) $\phi(p, T x) \leq \phi(p, x)$ for $x \in C$ and $p \in F(T)$, and (A3) $F(T)=\widehat{F}(T)$ and is said to be Bregman relatively nonexpansive with respect to $f$ if (B1) $F(T) \neq \emptyset$; (B2) $D_{f}(p, T x) \leq D_{f}(p, x)$ for $x \in C, p \in F(T)$ and (B3) $F(T)=\widehat{F}(T)$. We remark that the class of relatively nonexpansive mappings is contained in a class of Bregman relatively nonexpansive mappings with respect to $f(x)=\|x\|^{2}$.

Let $N(C)$ and $C B(C)$ denote the family of nonempty subsets and nonempty closed bounded subsets of $C$, respectively. Let $H$ be the Hausdorff metric on $C B(C)$ defined by

$$
H(A, B)=\max \left\{\sup _{a \in A} d(a, B), \sup _{b \in B} d(b, A)\right\}
$$

for all $A, B \in C B(C)$, where $d(a, B)=\inf \{\|a-b\|: b \in B\}$ is the distance from the point $a$ to the subset $B$.

Let $T: C \rightarrow C B(C)$ be a mapping. $T$ is said to be nonexpansive if $H(T x, T y) \leq\|x-y\|$, for all $x, y \in C$. An element $p \in C$ is called a fixed point of $T$, if $p \in F(T)$, where $F(T):=$ $\{p \in C: p \in T(p)\}$. A point $p \in C$ is called an asymptotic fixed point of $T$, if there exists a sequence $\left\{x_{n}\right\}$ in $C$ which converges weakly to $p$ such that $\lim _{n \rightarrow \infty} d\left(x_{n}, T x_{n}\right)=0 . T$ is called relatively nonexpansive if (A1) $F(T) \neq \emptyset$; (A2) $\phi(p, u) \leq \phi(p, x)$ for all $u \in T x, x \in C$, and (A3)' $F(T)=\widehat{F}(T)$. A mapping $T$ is called quasi-Bregman nonexpansive with respect to $f$ if $F(T) \neq \emptyset$ and $D_{f}(p, u) \leq D_{f}(p, x)$ for all $u \in T x, x \in C, p \in F(T)$ and is called Bregman relatively nonexpansive with respect to $f$ if (B1) $F(T) \neq \emptyset$; $(\mathrm{B} 2)^{\prime} D_{f}(p, u) \leq D_{f}(p, x)$ for $u \in T x, x \in C, p \in F(T)$, and $(\mathrm{B} 3)^{\prime} F(T)=\widehat{F}(T)$.

We note that the class of multi-valued relatively nonexpansive mappings is contained in a class of multi-valued Bregman relatively nonexpansive mappings which includes the class of single-valued Bregman relatively nonexpansive mappings. Hence, the class of multi-valued Bregman relatively nonexpansive mappings is a more general class of mappings. An example of a multi-valued Bregman relatively nonexpansive mapping is given now. 
Example 1.2 Let $I=[0,1], X=L^{p}(I), 1<p<\infty$ and $C=\{f \in X: f(x) \geq 0, \forall x \in I\}$. Let $T: C \rightarrow C B(C)$ be defined by

$$
T(f)= \begin{cases}\left\{h \in C: f(x)-\frac{1}{2} \leq h(x) \leq f(x)-\frac{1}{4}, \forall x \in I\right\}, & \text { if } f(x)>1, \forall x \in I \\ \{0\}, & \text { otherwise }\end{cases}
$$

Let $g: E \rightarrow \mathbb{R}$ be defined by $g(x)=\frac{1}{p}\|x\|^{p}, 1<p<\infty, x \in E$. Clearly, we have $\nabla g(x)=J_{p}(x)$ for all $x \in E$, and $g^{*}\left(x^{*}\right)=\frac{1}{q}\left\|x^{*}\right\|^{q}$, where $1<q<\infty$ satisfies $\frac{1}{p}+\frac{1}{q}=1$. It is clear that $F(T)=$ $\{0\}$. Let $f \in C$ and $h \in T(f)$ such that $f(x)>1$ for all $x \in[0,1]$. Then, using (1.3), we get

$$
\begin{aligned}
D_{g}(0, h) & =D_{g^{*}}(\nabla g(h), \nabla g(0))=D_{g^{*}}\left(J_{p}(h), 0\right) \\
& =g^{*}\left(J_{p}(h)\right)-g^{*}(0)-\left\langle\nabla g^{*}(0), J_{p}(h)-0\right\rangle \\
& =\frac{1}{q}\left\|J_{p}(h)\right\|^{q} \leq \frac{1}{q}\left\|J_{p}(f)\right\|^{q} \\
& =D_{g^{*}}\left(J_{p}(f), 0\right)=D_{g}(0, f) .
\end{aligned}
$$

Next, let $f \in C$ such that there exists $x \in I$ such that $f(x) \leq 1$, then

$$
D_{g}(0,0)=D_{g^{*}}(0,0) \leq D_{g^{*}}\left(J_{p}(f), 0\right)=D_{g}(0, f) .
$$

Hence, $T$ is a multi-valued quasi-Bregman nonexpansive mapping. Now, we show that $\widehat{F}(T)=F(T)$. Let $\left\{f_{n}\right\} \subset C$ be a sequence which converges weakly to $h$, and $z_{n}=$ $d\left(f_{n}, T\left(f_{n}\right)\right) \rightarrow 0$. Let $n \in \mathbb{N}$, then we have

$$
z_{n}= \begin{cases}\frac{1}{4}, & \text { if } f_{n}(x)>1, \forall x \in[0,1] \\ \left\|f_{n}\right\|_{p}, & \text { otherwise }\end{cases}
$$

Since $z_{n} \rightarrow 0$, we have $\left\|f_{n}\right\|_{p} \rightarrow 0$ and hence $h=0$. Therefore, $T$ is a multi-valued Bregman relatively nonexpansive mapping.

The approximations of fixed points of nonexpansive, quasi-nonexpansive, relatively nonexpansive, and relatively quasi-nonexpansive mappings when they exist have been intensively studied for almost 40 years or so by various authors (see, e.g., [8-18] and the references therein) in Banach spaces.

In 1967, Bregman [5] discovered an effective technique using the so-called Bregman distance function $D_{f}$ in the process of designing and analyzing feasibility and optimization algorithms. This opened a growing area of research in which Bregman's technique is applied in various ways in order to design and analyze iterative algorithms for solving not only feasibility and optimization problems, but also algorithms for solving variational inequality problems, equilibrium problems, fixed point problems for nonlinear mappings, and so on (see, e.g. $[7,19,20]$, and the references therein).

In [21], Reich and Sabach proposed the following algorithms for finding common fixed points of finitely many Bregman firmly nonexpansive operators defined on a nonempty, closed and convex subset $C$ of a reflexive Banach space $E$ (see also [22, 23]). The construction of fixed points for Bregman-type single-valued mappings via iterative processes has been investigated in, for example, [21, 24-27]. This now leads to the following important question. 
Question Is it possible to obtain the results of Reich and Sabach [21] for the class of multivalued Bregman relatively nonexpansive mappings?

The study of fixed points for multi-valued nonexpansive mappings using the Hausdorff metric was introduced by Markin [28] (see also [29]). Later, an interesting and rich fixed point theory for such mappings was developed which has applications in control theory, convex optimization, differential inclusion, and economics (see, for example, [30] and references therein). Moreover, the existence of fixed points for multi-valued nonexpansive mappings in uniformly convex Banach spaces was proved by Lim [31] (see also [32]).

Recently, Homaeipour and Razani [33] studied the following iterative scheme for a fixed point of relatively nonexpansive multi-valued mapping in uniformly convex and uniformly smooth Banach space $E$ :

$$
\left\{\begin{array}{l}
x_{0} \in C, \quad \text { chosen arbitrary, } \\
x_{n+1}=\Pi_{C} J^{-1}\left(\alpha_{n} J x_{n}+\left(1-\alpha_{n}\right) J z_{n}\right), \quad z_{n} \in T x_{n}, n \geq 0,
\end{array}\right.
$$

where $\left\{\alpha_{n}\right\} \subset(0,1)$ for all $n \geq 0$ and $\liminf _{n \rightarrow \infty} \alpha_{n}\left(1-\alpha_{n}\right)>0$. They proved that if $J$ is weakly sequentially continuous then the sequence $\left\{x_{n}\right\}$ converges weakly to a fixed point of $T$. Furthermore, it is shown that the scheme converges strongly to a fixed point of $T$ if the interior of $F(T)$ is nonempty.

More recently, Zegeye and Shahzad [34], extended the above result to a finite family of multi-valued relatively nonexpansive mappings without the assumption that the interior of $F(T)$ is nonempty. In fact, they proved that if $C$ is a nonempty, closed, and convex subset of a uniformly smooth and uniformly convex real Banach space $E$ and $T_{i}: C \rightarrow C B(C)$, for $i=1,2, \ldots, N$, are relatively nonexpansive multi-valued mappings with $\mathcal{F}:=\bigcap_{i=1}^{N} F\left(T_{i}\right)$ nonempty, then the sequence $\left\{x_{n}\right\}$ generated by

$$
\left\{\begin{array}{l}
x_{0}=w \in C, \quad \text { chosen arbitrarily, } \\
y_{n}=\Pi_{C} J^{-1}\left(\alpha_{n} J w+\left(1-\alpha_{n}\right) J x_{n}\right), \\
x_{n+1}=J^{-1}\left(\beta_{n, 0} J x_{n}+\sum_{i=1}^{N} \beta_{n, j} J u_{n, i}\right), \quad u_{n, i} \in T_{i} y_{n}, n \geq 0,
\end{array}\right.
$$

where $\alpha_{n} \in(0,1)$ and $\left\{\beta_{n, i}\right\} \subset[a, b] \subset(0,1)$, for $i=1,2, \ldots, N$, satisfy certain conditions, converges strongly to an element of $\mathcal{F}$.

In this paper, it is our purpose to introduce an iterative scheme which converges strongly to a common fixed point of a finite family of multi-valued Bregman relatively nonexpansive mappings. We prove strong convergence theorems for the sequences produced by the method. Our results improve and generalize many known results in the current literature (see, for example, $[33,34]$ ).

\section{Preliminaries}

Let $E$ be a reflexive real Banach space and $E^{*}$ as its dual. Let $f: E \rightarrow(-\infty,+\infty]$ be a Gâteaux differentiable function. The modulus of the total convexity of $f$ at $x \in \operatorname{dom} f$ is the function $v_{f}(x, \cdot):[0,+\infty) \rightarrow[0,+\infty]$ defined by

$$
v_{f}(x, t):=\inf \left\{D_{f}(y, x): y \in \operatorname{dom} f,\|y-x\|=t\right\} .
$$

The function $f$ is called totally convex at $x$ if $v_{f}(x, t)>0$, whenever $t>0$. The function $f$ is called totally convex if it is totally convex at any point $x \in \operatorname{int}(\operatorname{dom} f)$ and is said to be 
totally convex on bounded sets if $v_{f}(B, t)>0$ for any nonempty bounded subset $B$ of $E$ and $t>0$, where the modulus of total convexity of the function $f$ on the set $B$ is the function $v_{f}: \operatorname{int} \operatorname{dom} f \times[0,+\infty) \rightarrow[0,+\infty]$ defined by

$$
v_{f}(B, t):=\inf \left\{V_{f}(x, t): x \in B \cap \operatorname{dom} f\right\} .
$$

Let $E$ be a Banach space and let $B_{r}:=\{z \in E:\|z\| \leq r\}$ for all $r>0$ and $S_{E}=\{x \in E:\|x\|=$ $1\}$. Then a function $f: E \rightarrow \mathbb{R}$ is said to be uniformly convex on bounded subsets of $E$ [35, pp.203] if $\rho_{r}(t)>0$ for all $r, t>0$, where $\rho_{r}:[0, \infty) \rightarrow[0, \infty]$ is defined by

$$
\rho_{r}(t):=\inf _{x, y \in B_{r},\|x-y\|=t, \alpha \in(0,1)} \frac{\alpha f(x)+(1-\alpha) f(y)-f(\alpha x+(1-\alpha) y)}{\alpha(1-\alpha)},
$$

for all $t \geq 0$. The function $\rho_{r}$ is called the gauge of the uniform convexity of $f$. We know that $f$ is totally convex on bounded sets if and only if $f$ is uniformly convex on bounded sets (see [36], Theorem 2.10).

If $f$ is uniformly convex, then the following lemma is known.

Lemma 2.1 [37] Let $E$ be a Banach space, let $r>0$ be a constant, and let $f: E \rightarrow \mathbb{R}$ be a uniformly convex function on bounded subsets of $E$. Then

$$
f\left(\sum_{k=0}^{n} \alpha_{k} x_{k}\right) \leq \sum_{k=0}^{n} \alpha_{k} f\left(x_{k}\right)-\alpha_{i} \alpha_{j} \rho_{r}\left(\left\|x_{i}-y_{j}\right\|\right),
$$

for all $i, j \in\{0,1,2, \ldots, n\}, x_{k} \in B r, \alpha_{k} \in(0,1)$, and $k=0,1,2, \ldots, n$ with $\sum_{k=0}^{n} \alpha_{k}=1$, where $\rho_{r}$ is the gauge of uniform convexity of $f$.

A function $f$ on $E$ is coercive [38] if the sublevel set of $f$ is bounded; equivalently, $\lim _{\|x\| \rightarrow \infty} f(x)=\infty$. A function $f$ on $E$ is said to be strongly coercive [35] if $\lim _{\|x\| \rightarrow \infty} f(x) /$ $\|x\|=\infty$.

In the sequel, we shall need the following lemmas.

Lemma 2.2 [39] The function $f: E \rightarrow(-\infty,+\infty)$ is totally convex on bounded subsets of $E$ if and only iffor any two sequences $\left\{x_{n}\right\}$ and $\left\{y_{n}\right\}$ in $\operatorname{int}(\operatorname{dom} f)$ and $\operatorname{dom} f$, respectively, such that the first one is bounded, we have

$$
\lim _{n \rightarrow \infty} D_{f}\left(y_{n}, x_{n}\right)=0 \quad \Longrightarrow \quad \lim _{n \rightarrow \infty}\left\|y_{n}-x_{n}\right\|=0
$$

Lemma 2.3 [35] Let $f: E \rightarrow \mathbb{R}$ be a strongly coercive and uniformly convex on bounded subsets of $E$, then $f^{*}$ is bounded and uniformly Fréchet differentiable on bounded subsets of $E^{*}$.

Lemma 2.4 [26] Let $f: E \rightarrow(-\infty,+\infty]$ be a uniformly Fréchet differentiable and bounded on bounded sets of $E$, then $\nabla f$ is uniformly continuous on bounded subsets of $E$ from the strong topology of $E$ to the strong topology of $E^{*}$. 
Lemma 2.5 [1] Let $f: E \rightarrow(-\infty,+\infty$ ] be a proper, lower semicontinuous and convex function, then $f^{*}: E^{*} \rightarrow(-\infty,+\infty]$ is a proper, weak $k^{*}$ lower semicontinuous and convex function. Thus, for all $z \in E$, we have

$$
D_{f}\left(z, \nabla f^{*}\left(\sum_{i=1}^{N} t_{i} \nabla f\left(x_{i}\right)\right)\right) \leq \sum_{i=1}^{N} t_{i} D_{f}\left(z, x_{i}\right) .
$$

Lemma 2.6 [40] Let $f: E \rightarrow \mathbb{R}$ be a Gâteaux differentiable on $\operatorname{int}(\operatorname{dom} f)$ such that $\nabla f^{*}$ is bounded on bounded subsets of dom $f^{*}$. Let $x \in E$ and $\left\{x_{n}\right\} \subset E$. If $\left\{D_{f}\left(x, x_{n}\right)\right\}$ is bounded, so is the sequence $\left\{x_{n}\right\}$.

Lemma 2.7 [36] Let $C$ be a nonempty, closed, and convex subset of $E$. Let $f: E \rightarrow \mathbb{R}$ be a Gâteaux differentiable and totally convex function and let $x \in E$. Then

(i) $z=P_{C}^{f}(x)$ if and only if $\langle\nabla f(x)-\nabla f(z), y-z\rangle \leq 0, \forall y \in C$.

(ii) $D_{f}\left(y, P_{C}^{f}(x)\right)+D_{f}\left(P_{C}^{f}(x), x\right) \leq D_{f}(y, x), \forall y \in C$.

Let $f: E \rightarrow \mathbb{R}$ be a Legendre and Gâteaux differentiable function. Following [6] and [4], we make use of the function $V_{f}: E \times E^{*} \rightarrow[0,+\infty)$ associated with $f$, which is defined by

$$
V_{f}\left(x, x^{*}\right)=f(x)-\left\langle x, x^{*}\right\rangle+f^{*}\left(x^{*}\right), \quad \forall x \in E, x^{*} \in E^{*} .
$$

Then we observe that $V_{f}$ is nonnegative and

$$
V_{f}\left(x, x^{*}\right)=D_{f}\left(x, \nabla f^{*}\left(x^{*}\right)\right) \quad \text { for all } x \in E \text { and } x^{*} \in E^{*} \text {. }
$$

Moreover, by the subdifferential inequality,

$$
V_{f}\left(x, x^{*}\right)+\left\langle y^{*}, \nabla f^{*}\left(x^{*}\right)-x\right\rangle \leq V_{f}\left(x, x^{*}+y^{*}\right)
$$

$\forall x \in E$ and $x^{*}, y^{*} \in E^{*}$ (see [41]).

Lemma 2.8 [42] Let $\left\{a_{n}\right\}$ be a sequence of nonnegative real numbers satisfying the following relation:

$$
a_{n+1} \leq\left(1-\alpha_{n}\right) a_{n}+\alpha_{n} \delta_{n}, \quad n \geq n_{0},
$$

where $\left\{\alpha_{n}\right\} \subset(0,1)$ and $\left\{\delta_{n}\right\} \subset \mathbb{R}$ satisfy the following conditions: $\lim _{n \rightarrow \infty} \alpha_{n}=0, \sum_{n=1}^{\infty} \alpha_{n}=$ $\infty$, and $\lim \sup _{n \rightarrow \infty} \delta_{n} \leq 0$. Then $\lim _{n \rightarrow \infty} a_{n}=0$.

Lemma 2.9 [43] Let $\left\{a_{n}\right\}$ be sequences of real numbers such that there exists a subsequence $\left\{n_{i}\right\}$ of $\{n\}$ such that $a_{n_{i}}<a_{n_{i}+1}$ for all $i \in \mathbb{N}$. Then there exists an increasing sequence $\left\{m_{k}\right\} \subset$ $\mathbb{N}$ such that $m_{k} \rightarrow \infty$ and the following properties are satisfied by all (sufficiently large) numbers $k \in \mathbb{N}$ :

$$
a_{m_{k}} \leq a_{m_{k}+1} \text { and } a_{k} \leq a_{m_{k}+1}
$$

In fact, $m_{k}$ is the largest number $n$ in the set $\{1,2, \ldots, k\}$ such that the condition $a_{n} \leq a_{n+1}$ holds. 


\section{Main result}

In the sequel we shall use the following proposition.

Proposition 3.1 Let $f: E \rightarrow \mathbb{R}$ be a uniformly Fréchet differentiable and totally convex on bounded subsets of $E$. Let $C$ be a nonempty, closed, and convex subset of $\operatorname{int}(\operatorname{dom} f)$ and $T: C \rightarrow C B(C)$ be a Bregman relatively nonexpansive mapping. Then $F(T)$ is closed and convex.

Proof First, we show that $F(T)$ is closed. Let $\left\{x_{n}\right\}$ be a sequence in $F(T)$ such that $x_{n} \rightarrow x^{*}$. Since $T$ is Bregman relatively nonexpansive mapping, we have $D_{f}\left(x_{n}, u\right) \leq D_{f}\left(x_{n}, x^{*}\right)$, for all $u \in T x^{*}$ for all $n \in \mathbb{N}$. Therefore,

$$
\begin{aligned}
D_{f}\left(x^{*}, u\right) & =\lim _{n \rightarrow \infty} D_{f}\left(x_{n}, u\right) \\
& \leq \lim _{n \rightarrow \infty} D_{f}\left(x_{n}, x^{*}\right) \\
& =D_{f}\left(x^{*}, x^{*}\right)=0 .
\end{aligned}
$$

Thus, by Lemma 2.2 we obtain $x^{*}=u$. Hence, $x^{*} \in F(T)$ and $F(T)$ is closed. Next, we show that $F(T)$ is convex. Let $x, y \in F(T)$ and $p=t x+(1-t) y$ for $t \in(0,1)$. We show that $p \in F(T)$. Let $w \in T(p)$, then we have

$$
\begin{aligned}
D_{f}(p, w)= & f(p)-f(w)-\langle\nabla f(w), p-w\rangle \\
= & f(p)-f(w)-\langle\nabla f(w), t x+(1-t) y-w\rangle \\
= & f(p)+t D_{f}(x, w)+(1-t) D_{f}(y, w)-t f(x)-(1-t) f(y) \\
\leq & f(p)+t D_{f}(x, p)+(1-t) D_{f}(y, p)-t f(x)-(1-t) f(y) \\
\leq & f(p)+t[f(x)-f(p)-\langle\nabla f(p), x-p\rangle] \\
& +(1-t)[f(y)-f(p)-\langle\nabla f(p), y-p\rangle]-t f(x)-(1-t) f(y) \\
= & \langle\nabla f(p), t x+(1-t) y-p\rangle=0 .
\end{aligned}
$$

Thus, by Lemma 2.2 we get $p \in T(p)$. Hence, $p \in F(T)$ and $F(T)$ is convex. Therefore, $F(T)$ is closed and convex.

Theorem 3.2 Let $f: E \rightarrow \mathbb{R}$ be a strongly coercive Legendre function which is bounded, uniformly Fréchet differentiable and totally convex on bounded subsets of E. Let $C$ be a nonempty, closed and convex subset of $\operatorname{int}(\operatorname{dom} f)$ and $T_{i}: C \rightarrow C B(C)$, for $i=1,2, \ldots, N$, be a finite family of Bregman relatively nonexpansive mappings such that $\mathcal{F}:=\bigcap_{i=1}^{N} F\left(T_{i}\right)$ is nonempty. For $u, x_{0} \in C$ let $\left\{x_{n}\right\}$ be a sequence generated by

$$
\left\{\begin{array}{l}
w_{n}=P_{C}^{f} \nabla f^{*}\left(\alpha_{n} \nabla f(u)+\left(1-\alpha_{n}\right) \nabla f\left(x_{n}\right)\right), \\
x_{n+1}=\nabla f^{*}\left(\beta_{0} \nabla f\left(w_{n}\right)+\sum_{i=1}^{N} \beta_{i} \nabla f\left(u_{i, n}\right)\right), \quad u_{i, n} \in T_{i} w_{n}, \forall n \geq 0,
\end{array}\right.
$$

where $\left\{\alpha_{n}\right\} \subset(0,1)$ and $\left\{\beta_{i}\right\}_{i=0}^{N} \subset[c, d] \subset(0,1)$ satisfy $\lim _{n \rightarrow \infty} \alpha_{n}=0, \sum_{n=1}^{\infty} \alpha_{n}=\infty$ and $\sum_{i=0}^{N} \beta_{i}=1$. Then $\left\{x_{n}\right\}$ converges strongly to $p=P_{\mathcal{F}}^{f}(u)$. 
Proof Proposition 3.1 ensures that each $F\left(T_{i}\right)$, for $i \in\{1,2, \ldots, N\}$, and hence $\mathcal{F}$, is closed and convex. Thus, $P_{\mathcal{F}}^{f}$ is well defined. Let $p=P_{\mathcal{F}}^{f}(u)$. Then, from (3.2), Lemmas 2.7, 2.5, and the property of $D_{f}$, we get

$$
\begin{aligned}
D_{f}\left(p, w_{n}\right) & =D_{f}\left(p, P_{C}^{f} \nabla f^{*}\left(\alpha_{n} \nabla f(u)+\left(1-\alpha_{n}\right) \nabla f\left(x_{n}\right)\right)\right) \\
& \leq D_{f}\left(p, \nabla f^{*}\left(\alpha_{n} \nabla f(u)+\left(1-\alpha_{n}\right) \nabla f\left(x_{n}\right)\right)\right) \\
& =\alpha_{n} D_{f}(p, u)+\left(1-\alpha_{n}\right) D_{f}\left(p, x_{n}\right) .
\end{aligned}
$$

Moreover, from (3.2), (2.3), and (2.2) we get

$$
\begin{aligned}
D_{f}\left(p, x_{n+1}\right)= & D_{f}\left(p, \nabla f^{*}\left(\beta_{0} \nabla f\left(w_{n}\right)+\sum_{i=1}^{N} \beta_{i} \nabla f\left(u_{i, n}\right)\right)\right) \\
= & V_{f}\left(p, \beta_{0} \nabla f\left(w_{n}\right)+\sum_{i=1}^{N} \beta_{i} \nabla f\left(u_{i, n}\right)\right) \\
= & f(p)-\left\langle p, \beta_{0} \nabla f\left(w_{n}\right)+\sum_{i=1}^{N} \beta_{i} \nabla f\left(u_{i, n}\right)\right\rangle \\
& +f^{*}\left(\beta_{0} \nabla f\left(w_{n}\right)+\sum_{i=1}^{N} \beta_{i} \nabla f\left(u_{i, n}\right)\right) .
\end{aligned}
$$

Since $f$ is uniformly Fréchet differentiable function we find that $f$ is uniformly smooth and hence by Theorem 3.5.5 of [35] we find that $f^{*}$ is uniformly convex. This, with Lemma 2.1 and (3.3), gives

$$
\begin{aligned}
D_{f}\left(p, x_{n+1}\right) \leq & f(p)-\beta_{0}\left\langle p, \nabla f\left(w_{n}\right)\right\rangle-\sum_{i=1}^{N} \beta_{i}\left|p, \nabla f\left(u_{i, n}\right)\right\rangle \\
& +\beta_{0} f^{*}\left(\nabla f\left(w_{n}\right)\right)+\sum_{i=1}^{N} \beta_{i} f^{*}\left(\nabla f\left(u_{i, n}\right)\right) \\
& -\beta_{0} \beta_{i} \rho_{r}^{*}\left(\left\|\nabla f\left(w_{n}\right)-\nabla f\left(u_{i, n}\right)\right\|\right) \\
= & \beta_{0} V\left(p, \nabla f\left(w_{n}\right)\right)+\sum_{i=1}^{N} \beta_{i} V\left(p, \nabla f\left(u_{i, n}\right)\right) \\
& -\beta_{0} \beta_{i} \rho_{r}^{*}\left(\left\|\nabla f\left(w_{n}\right)-\nabla f\left(u_{i, n}\right)\right\|\right) \\
= & \beta_{0} D_{f}\left(p, w_{n}\right)+\sum_{i=1}^{N} \beta_{i} D_{f}\left(p, u_{i, n}\right) \\
& -\beta_{0} \beta_{i} \rho_{r}^{*}\left(\left\|\nabla f\left(w_{n}\right)-\nabla f\left(u_{i, n}\right)\right\|\right) \\
\leq & \beta_{0} D_{f}\left(p, w_{n}\right)+\sum_{i=1}^{N} \beta_{i} D_{f}\left(p, w_{n}\right)-\beta_{0} \beta_{i} \rho_{r}^{*}\left(\left\|\nabla f\left(w_{n}\right)-\nabla f\left(u_{i, n}\right)\right\|\right) \\
\leq & D_{f}\left(p, w_{n}\right)-\beta_{0} \beta_{i} \rho_{r}^{*}\left(\left\|\nabla f\left(w_{n}\right)-\nabla f\left(u_{i, n}\right)\right\|\right) \leq D_{f}\left(p, w_{n}\right) \\
\leq & \alpha_{n} D_{f}(p, u)+\left(1-\alpha_{n}\right) D_{f}\left(p, x_{n}\right),
\end{aligned}
$$


for each $i \in\{1,2, \ldots, N\}$. Thus, by induction,

$$
D_{f}\left(p, x_{n+1}\right) \leq \max \left\{D_{f}(p, u), D_{f}\left(p, x_{0}\right)\right\}, \quad \forall n \geq 0,
$$

which implies that $\left\{x_{n}\right\}$ is bounded. Furthermore, from (3.2), (2.3), (2.4), and Lemma 2.7 we obtain

$$
\begin{aligned}
D_{f}\left(p, w_{n}\right)= & D_{f}\left(p, P_{C}^{f} \nabla f^{*}\left(\alpha_{n} \nabla f(u)+\left(1-\alpha_{n}\right) \nabla f\left(x_{n}\right)\right)\right) \\
\leq & D_{f}\left(p, \nabla f^{*}\left(\alpha_{n} \nabla f(u)+\left(1-\alpha_{n}\right) \nabla f\left(x_{n}\right)\right)\right) \\
= & V_{f}\left(p, \alpha_{n} \nabla f(u)+\left(1-\alpha_{n}\right) \nabla f\left(x_{n}\right)\right) \\
\leq & V_{f}\left(p, \alpha_{n} \nabla f(u)+\left(1-\alpha_{n}\right) \nabla f\left(x_{n}\right)-\alpha_{n}(\nabla f(u)-\nabla f(p))\right) \\
& +\alpha_{n}\left\langle\nabla f(u)-\nabla f(p), w_{n}-p\right\rangle \\
= & V_{f}\left(p, \alpha_{n} \nabla f(p)+\left(1-\alpha_{n}\right) \nabla f\left(x_{n}\right)\right) \\
& +\alpha_{n}\left\langle\nabla f(u)-\nabla f(p), w_{n}-p\right\rangle \\
= & D_{f}\left(p, \nabla f^{*}\left(\alpha_{n} \nabla f(p)+\left(1-\alpha_{n}\right) \nabla f\left(x_{n}\right)\right)\right) \\
& +\alpha_{n}\left\langle\nabla f(u)-\nabla f(p), w_{n}-p\right\rangle \\
\leq & \alpha_{n} D_{f}(p, p)+\left(1-\alpha_{n}\right) D_{f}\left(p, x_{n}\right) \\
& +\alpha_{n}\left\langle\nabla f(u)-\nabla f(p), w_{n}-p\right\rangle \\
= & \left(1-\alpha_{n}\right) D_{f}\left(p, x_{n}\right)+\alpha_{n}\left\langle\nabla f(u)-\nabla f(p), w_{n}-p\right\rangle .
\end{aligned}
$$

Furthermore, from (3.4) and (3.6) we have

$$
\begin{aligned}
D_{f}\left(p, x_{n+1}\right) \leq & \left(1-\alpha_{n}\right) D_{f}\left(p, x_{n}\right)+\alpha_{n}\left\langle\nabla f(u)-\nabla f(p), w_{n}-p\right\rangle \\
& -\beta_{0} \beta_{i} \rho_{r}^{*}\left(\left\|\nabla f\left(w_{n}\right)-\nabla f\left(u_{i, n}\right)\right\|\right) \\
\leq & \left(1-\alpha_{n}\right) D_{f}\left(p, x_{n}\right)+\alpha_{n}\left\langle\nabla f(u)-\nabla f(p), w_{n}-p\right\rangle .
\end{aligned}
$$

Now, we consider two cases.

Case 1. Suppose that there exists $n_{0} \in \mathbb{N}$ such that $\left\{D_{f}\left(p, x_{n}\right)\right\}$ is non-increasing for all $n \geq n_{0}$. In this situation, $\left\{D_{f}\left(p, x_{n}\right)\right\}$ is convergent. Then, from (3.7), we have

$$
\beta_{0} \beta_{i} \rho_{r}^{*}\left(\left\|\nabla f\left(w_{n}\right)-\nabla f\left(u_{i, n}\right)\right\|\right) \rightarrow 0,
$$

which implies, by the property of $\rho_{r}^{*}$ that

$$
\nabla f\left(w_{n}\right)-\nabla f\left(u_{i, n}\right) \rightarrow 0 \quad \text { as } n \rightarrow \infty .
$$

Now, since $f$ is strongly coercive and uniformly convex on bounded subsets of $E$ by Lemma 2.3 we see that $f^{*}$ is uniformly Fréchet differentiable on bounded subsets of $E^{*}$ and since $f$ is Legendre by Lemma 2.4 we find that $\nabla f^{*}$ is uniformly continuous on bounded subsets of $E^{*}$ and hence from (3.10) we get

$$
w_{n}-u_{i, n} \rightarrow 0 \quad \text { as } n \rightarrow \infty .
$$


In addition, since $d\left(w_{n}, T_{i} w_{n}\right) \leq\left\|w_{n}-u_{i, n}\right\|$ we have

$$
d\left(w_{n}, T_{i} w_{n}\right) \rightarrow 0 \quad \text { as } n \rightarrow \infty,
$$

for each $i \in\{1,2, \ldots, N\}$. Since $\left\{w_{n}\right\}$ is bounded and $E$ is reflexive, we choose a subsequence $\left\{w_{n_{j}}\right\}$ of $\left\{w_{n}\right\}$ such that $w_{n_{j}} \rightarrow w$ and $\limsup _{n \rightarrow \infty}\left\langle\nabla f(u)-\nabla f(p), w_{n}-p\right\rangle=\lim _{j \rightarrow \infty}\langle\nabla f(u)-$ $\left.\nabla f(p), w_{n_{j}}-p\right\rangle$. Thus, from (3.12) and the fact that each $T_{i}$ is Bregman relatively nonexpansive mapping we obtain $w \in F\left(T_{i}\right)$, for each $i \in\{1,2, \ldots, N\}$ and hence $w \in \bigcap_{i=1}^{N} F\left(T_{i}\right)$.

Therefore, by Lemma 2.7 , we immediately obtain

$$
\begin{aligned}
\limsup _{n \rightarrow \infty}\left\langle\nabla f(u)-\nabla f(p), w_{n}-p\right\rangle & =\lim _{j \rightarrow \infty}\left\langle\nabla f(u)-\nabla f(p), w_{n_{j}}-p\right\rangle \\
& =\langle\nabla f(u)-\nabla f(p), w-p\rangle \leq 0 .
\end{aligned}
$$

It follows from Lemma 2.8 and (3.8) that $D_{f}\left(p, x_{n}\right) \rightarrow 0$ as $n \rightarrow \infty$. Consequently, by Lemma 2.2 we obtain $x_{n} \rightarrow p \in \mathcal{F}$.

Case 2. Suppose that there exists a subsequence $\left\{n_{l}\right\}$ of $\{n\}$ such that

$$
D_{f}\left(p, x_{n_{l}}\right)<D_{f}\left(p, x_{n_{l}+1}\right)
$$

for all $l \in \mathbb{N}$. Then, by Lemma 2.9, there exists a nondecreasing sequence $\left\{m_{k}\right\} \subset \mathbb{N}$ such that $m_{k} \rightarrow \infty, D_{f}\left(p, x_{m_{k}}\right) \leq D_{f}\left(p, x_{m_{k}+1}\right)$, and $D_{f}\left(p, x_{k}\right) \leq D_{f}\left(p, x_{m_{k}+1}\right)$, for all $k \in \mathbb{N}$. Then, from (3.7) and the fact that $\alpha_{n} \rightarrow 0$, we obtain

$$
\rho_{r}^{*}\left(\left\|\nabla f\left(w_{m_{k}}\right)-\nabla f\left(u_{i, n_{k}}\right)\right\|\right) \rightarrow 0 \quad \text { as } k \rightarrow \infty,
$$

for each $i \in\{1,2, \ldots, N\}$. Thus, following the method of proof of Case 1, we obtain $d\left(w_{m_{k}}, T_{i} w_{m_{k}}\right) \rightarrow 0$ as $k \rightarrow \infty$, and hence we obtain

$$
\limsup _{k \rightarrow \infty}\left\langle\nabla f(u)-\nabla f(p), w_{m_{k}}-p\right\rangle \leq 0
$$

Then, from (3.8), we get

$$
D_{f}\left(p, x_{m_{k}+1}\right) \leq\left(1-\alpha_{m_{k}}\right) D_{f}\left(p, x_{m_{k}}\right)+\alpha_{m_{k}}\left\langle\nabla f(u)-\nabla f(p), w_{m_{k}}-p\right\rangle .
$$

Now, since $D_{f}\left(p, x_{m_{k}}\right) \leq D_{f}\left(p, x_{m_{k}+1}\right)$, inequality (3.14) implies that

$$
\begin{aligned}
\alpha_{m_{k}} D_{f}\left(p, x_{m_{k}}\right) \leq & D_{f}\left(p, x_{m_{k}}\right)-D_{f}\left(p, x_{m_{k}+1}\right) \\
& +\alpha_{m_{k}}\left\langle\nabla f(u)-\nabla f(p), w_{m_{k}}-p\right\rangle \\
\leq & \alpha_{m_{k}}\left\langle\nabla f(u)-\nabla f(p), w_{m_{k}}-p\right\rangle .
\end{aligned}
$$

Thus, we get

$$
D_{f}\left(p, x_{m_{k}}\right) \leq\left\langle\nabla f(u)-\nabla f(p), w_{m_{k}}-p\right\rangle
$$


Then, from (3.15) and (3.13), we obtain $D_{f}\left(p, x_{m_{k}}\right) \rightarrow 0$ as $k \rightarrow \infty$. This, together with (3.14), gives $D_{f}\left(p, x_{m_{k}+1}\right) \rightarrow 0$ as $k \rightarrow \infty$. But $D_{f}\left(p, x_{k}\right) \leq D_{f}\left(p, x_{m_{k}+1}\right)$ for all $k \in \mathbb{N}$, and hence we obtain $x_{k} \rightarrow p \in \mathcal{F}$. Therefore, from the above two cases, we can conclude that $\left\{x_{n}\right\}$ converges strongly to $p=P_{\mathcal{F}}^{f}(u)$ and the proof is complete.

If in Theorem 3.2, we assume that $N=1$, then we get the following corollary.

Corollary 3.3 Let $f: E \rightarrow \mathbb{R}$ be a strongly coercive Legendre function which is bounded, uniformly Fréchet differentiable and totally convex on bounded subsets of $E$. Let $C$ be a nonempty, closed, and convex subset of $\operatorname{int}(\operatorname{dom} f)$ and $T: C \rightarrow C B(C)$ be a Bregman relatively nonexpansive mapping such that $F(T)$ is nonempty. For $u, x_{0} \in C$ let $\left\{x_{n}\right\}$ be a sequence generated by

$$
\left\{\begin{array}{l}
w_{n}=P_{C}^{f} \nabla f^{*}\left(\alpha_{n} \nabla f(u)+\left(1-\alpha_{n}\right) \nabla f\left(x_{n}\right)\right), \\
x_{n+1}=\nabla f^{*}\left(\beta \nabla f\left(w_{n}\right)+(1-\beta) \nabla f\left(u_{n}\right)\right), \quad u_{n} \in T w_{n}, \forall n \geq 0,
\end{array}\right.
$$

where $\left\{\alpha_{n}\right\} \subset(0,1)$ and $\beta \in(0,1)$ satisfy $\lim _{n \rightarrow \infty} \alpha_{n}=0, \sum_{n=1}^{\infty} \alpha_{n}=\infty$. Then $\left\{x_{n}\right\}$ converges strongly to $p=P_{\mathcal{F}}^{f}(u)$.

If, in Theorem 3.2, we assume that each $T_{i}, i=1,2, \ldots, N$ is a single-valued Bregman relatively nonexpansive mapping, we get the following corollary.

Corollary 3.4 Let $f: E \rightarrow \mathbb{R}$ be a strongly coercive Legendre function which is bounded, uniformly Fréchet differentiable and totally convex on bounded subsets of E. Let $C$ be a nonempty, closed and convex subset of $\operatorname{int}(\operatorname{dom} f)$ and $T_{i}: C \rightarrow C$, for $i=1,2, \ldots, N$, be a finite family of Bregman relatively nonexpansive mappings such that $\mathcal{F}:=\bigcap_{i=1}^{N} F\left(T_{i}\right)$ is nonempty. For $u, x_{0} \in C$ let $\left\{x_{n}\right\}$ be a sequence generated by

$$
\left\{\begin{array}{l}
w_{n}=P_{C}^{f} \nabla f^{*}\left(\alpha_{n} \nabla f(u)+\left(1-\alpha_{n}\right) \nabla f\left(x_{n}\right)\right), \\
x_{n+1}=\nabla f^{*}\left(\beta_{0} \nabla f\left(w_{n}\right)+\sum_{i=1}^{N} \beta_{i} \nabla f\left(T_{i} w_{n}\right)\right), \quad \forall n \geq 0,
\end{array}\right.
$$

where $\left\{\alpha_{n}\right\} \subset(0,1)$ and $\left\{\beta_{i}\right\}_{i=0}^{N} \subset[c, d] \subset(0,1)$ satisfy $\lim _{n \rightarrow \infty} \alpha_{n}=0, \sum_{n=1}^{\infty} \alpha_{n}=\infty$ and $\sum_{i=0}^{N} \beta_{i}=1$. Then $\left\{x_{n}\right\}$ converges strongly to $p=P_{\mathcal{F}}^{f}(u)$.

If, in Theorem 3.2, we assume that each $T_{i}, i=1,2, \ldots, N$, is a multi-valued quasiBregman relatively nonexpansive mapping, we get the following corollary.

Corollary 3.5 Let $f: E \rightarrow \mathbb{R}$ be a strongly coercive Legendre function which is bounded, uniformly Fréchet differentiable, and totally convex on bounded subsets of E. Let $C$ be a nonempty, closed, and convex subset of $\operatorname{int}(\operatorname{dom} f)$ and $T_{i}: C \rightarrow C B(C)$, for $i=1,2, \ldots, N$, be a finite family of quasi-Bregman nonexpansive mappings with $F\left(T_{i}\right)=\widehat{F}\left(T_{i}\right)$, for each $i \in$ $\{1,2, \ldots, N\}$. Suppose that $\mathcal{F}:=\bigcap_{i=1}^{N} F\left(T_{i}\right)$ is nonempty. For $u, x_{0} \in C$ let $\left\{x_{n}\right\}$ be a sequence generated by

$$
\left\{\begin{array}{l}
w_{n}=P_{C}^{f} \nabla f^{*}\left(\alpha_{n} \nabla f(u)+\left(1-\alpha_{n}\right) \nabla f\left(x_{n}\right)\right), \\
x_{n+1}=\nabla f^{*}\left(\beta_{0} \nabla f\left(w_{n}\right)+\sum_{i=1}^{N} \beta_{i} \nabla f\left(u_{i, n}\right)\right), u_{i, n} \in T_{i} w_{n}, \quad \forall n \geq 0,
\end{array}\right.
$$


where $\left\{\alpha_{n}\right\} \subset(0,1)$ and $\left\{\beta_{i}\right\}_{i=0}^{N} \subset[c, d] \subset(0,1)$ satisfy $\lim _{n \rightarrow \infty} \alpha_{n}=0, \sum_{n=1}^{\infty} \alpha_{n}=\infty$ and $\sum_{i=0}^{N} \beta_{i}=1$. Then $\left\{x_{n}\right\}$ converges strongly to $p=D_{\mathcal{F}}^{f}(u)$.

Remark 3.6 (i) Theorem 3.2 improves and extends the corresponding results of Homaeipour and Razani [33] and Zegeye and Shahzad [34] to the class of multi-valued Bregman relatively nonexpansive mappings in a reflexive real Banach spaces. (ii) The requirement that the interior of $F$ is nonempty is dispensed with.

\section{Competing interests}

The authors declare that they have no competing interests.

\section{Authors' contributions}

All authors contributed equally to the writing of this paper. All authors read and approved the final manuscript.

\section{Author details}

${ }^{1}$ Department of Mathematics, King Abdulaziz University, P.O. Box 80203, Jeddah, 21589, Saudi Arabia. ${ }^{2}$ Department of Mathematics, University of Botswana, Pvt. Bag 00704, Gaborone, Botswana.

\section{Acknowledgements}

This article was funded by the Deanship of Scientific Research (DSR), King Abdulaziz University, Jeddah. The first author acknowledges with thanks DSR for financial support. The authors are grateful to the anonymous reviewers for useful comments

Received: 24 February 2014 Accepted: 27 June 2014 Published: 22 Jul 2014

\section{References}

1. Phelps, RP: Convex Functions, Monotone Operators, and Differentiability, 2nd edn. Lecture Notes in Mathematics, vol. 1364. Springer, Berlin (1993)

2. Bauschke, HH, Borwein, JM, Combettes, PL: Essential smoothness, essential strict convexity, and Legendre functions in Banach spaces. Commun. Contemp. Math. 3, 615-647 (2001)

3. Bonnans, JF, Shapiro, A: Perturbation Analysis of Optimization Problems. Springer, New York (2000)

4. Censor, Y, Lent, A: An iterative row-action method for interval convex programming. J. Optim. Theory Appl. 34, 321-353 (1981)

5. Bregman, LM: The relaxation method for finding the common point of convex sets and its application to the solution of problems in convex programming. USSR Comput. Math. Math. Phys. 7, 200-217 (1967)

6. Alber, Yl: Metric and generalized projection operators in Banach spaces: properties and applications. In: Theory and Applications of Nonlinear Operators of Accretive and Monotone Type. Lect. Notes Pure Appl. Math., vol. 178, pp. 15-50 (1996)

7. Reich, S: A weak convergence theorem for the alternating method with Bergman distance. In: Kartsatos, AG (ed.) Theory and Applications of Nonlinear Operators of Accretive and Monotone Type. Lect. Notes Pure Appl. Math., vol. 178, pp. 313-318. Dekker, New York (1996)

8. Browder, FE: Convergence of approximants to fixed points of nonexpansive nonlinear mappings in Banach spaces. Arch. Ration. Mech. Anal. 24, 82-90 (1967)

9. Reich, S: Strong convergence theorems for resolvents of accretive operators in Banach spaces. J. Math. Anal. Appl. 75, 287-292 (1980)

10. Habtu, H, Shahzad, N: Viscosity approximation methods for a common fixed point of finite family of nonexpansive mappings. Appl. Math. Comput. 191(1), 155-163 (2007)

11. Zegeye, $\mathrm{H}$, Shahzad, N: Approximation methods for a common fixed point of finite family of nonexpansive mappings. Numer. Funct. Anal. Optim. 28(11-12), 1405-1419 (2007)

12. Zegeye, $\mathrm{H}$, Shahzad, $\mathrm{N}$ : Viscosity approximation methods for a common fixed point of a family of quasi-nonexpansive mappings. Nonlinear Anal. 68, 2005-2012 (2008)

13. Zegeye, $\mathrm{H}$, Shahzad, $\mathrm{N}$ : Strong convergence theorems for a finite family of nonexpansive mappings and semi-groups via the hybrid method. Nonlinear Anal. (2009). doi:10.1016/j.na.2009.06.056

14. Zegeye, $\mathrm{H}$, Shahzad, $\mathrm{N}$ : A hybrid scheme for finite families of equilibrium, variational inequality and fixed point problems. Nonlinear Anal. 74, 263-272 (2011)

15. Zegeye, $\mathrm{H}$, Shahzad, N: Strong convergence for monotone mappings and relatively weak nonexpansive mappings. Nonlinear Anal. 70, 2707-2716 (2009)

16. Zegeye, $\mathrm{H}$, Ofoedu, EU, Shahzad, N: Convergence theorems for equilibrium problem, variational inequality problem and countably infinite relatively quasi-nonexpansive mappings. Nonlinear Anal. 216, 3439-3449 (2010)

17. Zegeye, H, Shahzad, N, Alghamdi, MA: Minimum-norm fixed point of pseudocontractive mappings. Abstr. Appl. Anal. 2012, Article ID 926017 (2012)

18. Zegeye, H, Shahzad, N, Alghamdi, MA: Convergence of Ishikawa's iteration method for pseudocontractive mappings. Nonlinear Anal. 74, 7304-7311 (2011)

19. Bruck, RE, Reich, S: Nonexpansive projections and resolvents of accretive operators in Banach spaces. Houst. J. Math 3, 459-470 (1977) 
20. Reich, S, Sabach, S: Two strong convergence theorems for Bregman strongly nonexpansive operators in reflexive Banach spaces. Nonlinear Anal. 73, 122-135 (2010)

21. Reich, S, Sabach, S: A projection method for solving nonlinear problems in reflexive Banach spaces. J. Fixed Point Theory Appl. 9, 101-116 (2011)

22. Reich, S: A limit theorem for projections. Linear Multilinear Algebra 13, 281-290 (1983)

23. Censor, Y, Reich, S: Iterations of paracontractions and firmly nonexpansive operators with applications to feasibility and optimization. Optimization 37, 323-339 (1996)

24. Bauschke, $\mathrm{HH}$, Combettes, PL: Construction of best Bregman approximations in reflexive Banach spaces. Proc. Am. Math. Soc. 131, 3757-3766 (2003)

25. Shahzad, N, Zegeye, H, Alotaibi, A: Convergence results for a common solution of a finite family of variational inequality problems for monotone mappings with Bregman distance function. Fixed Point Theory Appl. 2013, Article ID 343 (2013)

26. Reich, S, Sabach, S: A strong convergence theorem for a proximal-type algorithm in reflexive Banach spaces. J. Nonlinear Convex Anal. 10, 471-485 (2009)

27. Reich, S, Sabach, S: Two strong convergence theorems for a proximal method in reflexive Banach spaces. Numer. Funct. Anal. Optim. 31, 22-44 (2010)

28. Markin, JT: Continuous dependence of fixed point sets. Proc. Am. Math. Soc. 38, 545-547 (1973)

29. Nadler, SB: Multi-valued contraction mappings. Pac. J. Math. 30, 475-488 (1969)

30. Gorniewicz, L: Topological Fixed Point Theory of Multi-Valued Mappings. Kluwer Academic, Dordrecht (1999)

31. Lim, TC: A fixed point theorem for multi-valued nonexpansive mappings in a uniformly convex Banach spaces. Bull. Am. Math. Soc. 80, 1123-1126 (1974)

32. Reich, S: Approximate selections, best approximations, fixed points, and invariant sets. J. Math. Anal. Appl. 62, 104-113 (1978)

33. Homaeipour, S, Razani, A: Weak and strong convergence theorems for relatively nonexpansive multi-valued mappings in Banach spaces. Fixed Point Theory Appl. 2011, Article ID 73 (2011). doi:10.1186/1687-1812-2011-73

34. Zegeye, $\mathrm{H}$, Shahzad, $\mathrm{N}$ : Convergence theorems for a common point of solutions of equilibrium and fixed point of relatively nonexpansive multi-valued mapping problems. Abstr. Appl. Anal. 2012, Article ID 859598 (2012)

35. Zalinescu, C: Convex Analysis in General Vector Spaces. World Scientific, River Edge (2002)

36. Butnariu, D, Resmerita, E: Bregman distances, totally convex functions and a method for solving operator equations in Banach spaces. Abstr. Appl. Anal. 2006, Article ID 84919 (2006)

37. Naraghirad, E, Yao, J-C: Bregman weak relatively nonexpansive mappings in Banach spaces. Fixed Point Theory Appl. 2013, Article ID 141 (2013). doi:10.1186/1687-1812-2013-141

38. Hiriart-Urruty, J-B, Lemarchal, C: Convex Analysis and Minimization Algorithms II. Grundlehren der mathematischen Wissenschaften, vol. 306. Springer, Berlin (1993)

39. Butnariu, D, lusem, AN: Totally Convex Functions for Fixed Points Computation and Infinite Dimensional Optimization. Kluwer Academic, Dordrecht (2000)

40. Martin-Marqueza, V, Reich, S, Sabach, S: Bregman strongly nonexpansive operators in reflexive Banach spaces. J. Math. Anal. Appl. 400, 597-614 (2013)

41. Kohsaka, F, Takahashi, W: Proximal point algorithms with Bregman functions in Banach spaces. J. Nonlinear Convex Anal. 6, 505-523 (2005)

42. Xu, HK: Another control condition in an iterative method for nonexpansive mappings. Bull. Aust. Math. Soc. 65 109-113 (2002)

43. Maingé, PE: Strong convergence of projected subgradient methods for nonsmooth and nonstrictly convex minimization. Set-Valued Anal. 16, 899-912 (2008)

10.1186/1687-1812-2014-152

Cite this article as: Shahzad and Zegeye: Convergence theorem for common fixed points of a finite family of multi-valued Bregman relatively nonexpansive mappings. Fixed Point Theory and Applications 2014, 2014:152

\section{Submit your manuscript to a SpringerOpen ${ }^{\circ}$ journal and benefit from:}

- Convenient online submission

Rigorous peer review

- Immediate publication on acceptance

- Open access: articles freely available online

- High visibility within the field

- Retaining the copyright to your article 